\section{Association Between Fasting Glucose Variability in Young Adulthood and the Progression of Coronary Artery Calcification in Middle Age}

Diabetes Care 2020;43:2574-2580 | https://doi.org/10.2337/dc20-0838

\section{OBJECTIVE}

To investigate whether intraindividual variability of fasting glucose (FG) in young adulthood is associated with coronary artery calcification (CAC) progression in middle age.

\section{RESEARCH DESIGN AND METHODS}

We included 2,256 CARDIA (Coronary Artery Risk Development Study in Young Adults) participants with CAC assessment by computed tomography scanner at baseline (2000-2001) and 10 years later (2010-2011). CAC progression was assessed for each individual as the difference of logarithmic CAC scores at follow-up and baseline ( $\log [$ CAC (follow-up) + 1] - $\log [$ CAC (baseline) + 1]). FG variability was defined by the coefficient of variation about the mean FG (FG-CV), the SD of FG (FGSD), and the average real variability of FG (FG-ARV) during the 10-year follow-up. We investigated the association between FG variability and CAC progression with adjustment for demographics, clinical risk factors, mean FG level, change in FG level, diabetes incidence, and medication use.

\section{RESULTS}

After multivariable adjustment, 1-SD increment in FG-CV was associated with worse progression of CAC as demonstrated as percent change in CAC, with incident CAC $5.9 \%(95 \% \mathrm{Cl} 1.0,10.7)$ and any CAC progression $6.7 \%(95 \% \mathrm{Cl} 2.3,11.1)$ during 10 years. Similar findings were also observed in FG-SD and FG-ARV.

\section{CONCLUSIONS}

Higher FG variability during young adulthood was associated with greater CAC progression in middle age, suggesting its value in predicting risk for subclinical coronary artery diseases.

Individuals with diabetes have at least a twofold increased risk of cardiovascular events compared with those without diabetes, and cardiovascular events are the leading cause of diabetes-related morbidity and mortality (1-3). Coronary artery calcification (CAC), a noninvasive marker of subclinical atherosclerosis, has been identified to be an independent predictor of cardiovascular events (4-7). The American Diabetes Association recommends CAC screening as cardiovascular risk assessment in adults with diabetes $\geq 40$ years of age (8). Due to the rapidly increasing
Weijing Feng, ${ }^{1,2,3}$ Zhibin $\mathrm{Li}^{4}{ }^{4}$

Wenjie Guo, ${ }^{1,2,3}$ Xianglin Fan, ${ }^{1,2,3}$

Feiran Zhou, ${ }^{1,2,3}$ Kun Zhang, ${ }^{4}$

Caiwen Ou, ${ }^{1,2,3}$ Feifei Huang, ${ }^{4}$ and

Minsheng Chen ${ }^{1,2,3}$
${ }^{1}$ Department of Cardiology, Laboratory of Heart Center, Zhujiang Hospital, Southern Medical University, Guangzhou, China

${ }^{2}$ Guangdong Provincial Key Laboratory of Shock and Microcirculation, Guangzhou, China

${ }^{3}$ Sino-Japanese Cooperation Platform for Translational Research in Heart Failure, Guangzhou, China

${ }^{4}$ Department of Cardiology, Sun Yat-sen Memorial Hospital, Sun Yat-sen University, Guangzhou, China

Corresponding author: Minsheng Chen, gzminsheng@ vip.163.com, Caiwen Ou, oucaiwen@smu.edu.cn or Feifei Huang, beier504@163.com

Received 14 April 2020 and accepted 25 June 2020

This article contains supplementary material online at https://doi.org/10.2337/figshare.12588878.

(C) 2020 by the American Diabetes Association. Readers may use this article as long as the work is properly cited, the use is educational and not for profit, and the work is not altered. More information is available at https://www.diabetesjournals .org/content/license. 
prevalence of prediabetes and diabetes worldwide, there is an urgent need to understand the impact of diabetes-related factors on cardiovascular structure and function in the early disease process (9).

Recent epidemiological evidence suggests that greater long-term variability in plasma glucose is associated with higher risk for cardiovascular events among individual with diabetes (10-13). Whether this association is attributable to the increase of average plasma glucose level, variability due to diabetes medication use, or other cumulative risk factors is unclear. Moreover, the association between glucose variability and CAC has not been reported. Investigating the contribution of variability of glucose among young adults and adults in early middle age without diabetes may advance our understanding of how dysfunction in glucose homeostasis impacts CAC progression, a subclinical cardiovascular disease in early life. Our objective was to examine the prospective association of fasting glucose (FG) variability with 10-year CAC progression. We hypothesized that greater FG variability during young adulthood would be associated with advanced CAC progression by middle age.

\section{RESEARCH DESIGN AND METHODS}

\section{Study Population}

The Coronary Artery Risk Development Study in Young Adults (CARDIA) is a multicenter prospective investigation of 5,115 healthy young adults aged 18-30 years recruited in 1985-1986 from four U.S. metropolitan communities. A detailed design of CARDIA has previously been published (14). Participants are invited to participate in follow-up examinations approximately every $2-5$ years. In this current study, data from follow-up examinations at CARDIA years 15 (20002001), 20 (2005-2006), and 25 (20102011) were collected. Participant retention rate was $74 \%, 72 \%$, and $72 \%$ at year 15 , 20 , and 25 , respectively, of the surviving participants. All participants provided written informed consent at each examination, and the institutional review board at each study site and coordinating center approved the study procedure for all examinations.

For our analysis, we excluded participants who did not have information for any of the CAC assessments at years
15 and 25 ( $n=456$ ) or had fewer than three valid FG values from year 15 to year 25 exams ( $n=786)$. The final sample size for the analysis of any CAC progression was 2,256 participants (Supplementary Fig. 1). For incident CAC, those without baseline CAC at the year 15 examination ( $n=2,062$ ) were included for that analysis.

\section{FG Measurement}

FG was measured in nonpregnant participants who reported fasting $\geq 8 \mathrm{~h}$ at years 15, 20, and 25. FG was assayed using hexokinase coupled to glucose6-phosphate dehydrogenase (Linco Research, St Louis, MO). Quality control using a commercially purchased pool of control subjects showed that withinrun precision and between-run precision is $<1 \%$ and $<2 \%$ coefficient of variation (CV), respectively (15). The data were recalibrated to standardize serum glucose values across CARDIA examinations.

\section{Covariate Ascertainment}

Standardized questionnaires and protocols were used to collect data on participant demographic characteristics, smoking, alcohol, physical activity, medical history, and use of medications (14). Height and weight were measured without shoes and in light clothing and were used to calculate BMI (weight in kilograms divided by the square of height in meters). Blood pressure (BP) was measured in triplicate after a 5-min rest using either a random-zero mercury sphygmomanometer (examination year 15) or an automated oscillometer (examination years 20 and 25) (model HEM907XL; OMRON). Values from the oscillometer were calibrated to the sphygmomanometric measures across examinations. BP was determined as the mean of the second and third measurements (16). Other laboratory measures, such as serum creatinine, total cholesterol, and LDL cholesterol (LDL-C), were collected for analysis.

\section{CAC Assessment}

CAC was assessed using electron beam computed tomography (Chicago and Oakland centers at years 15 and 20) or multidetector computed tomography scanners (Birmingham and Minneapolis centers at years 15 and 20 and all centers at year 25). A standardized protocol for the CAC assessment process has previously been described in detail (17). Contiguous 3-mm-thick slices from the root of the aorta to the apex of the heart were obtained. Image data were transmitted electronically to the CARDIA Reading Center, and a trained technician blinded to participant characteristics identified a region of interest for each potential foci of CAC. A calcium score in Agatston units was calculated for each calcified lesion, and scores from the four major coronary arteries (left main, left circumflex, left anterior descending, and right coronary) were summed to compute a total calcium score (18). All image data were analyzed with high betweenreader and within-reader reproducibility (17).

\section{Statistical Analysis}

Continuous variables are described as mean (SD) for normally distributed data or median (range) for nonnormally distributed data. Categorical variables are reported as frequency (percentage). The main parameters of intraindividual FG variability were calculated for each participant between three successive FG measurements: the SD of FG (FG-SD), the CV of the mean FG (FG-CV), and the average real variability (ARV) of FG (FGARV). An FG variability formula has previously been published for CARDIA (15). CAC progression was computed for each individual as the difference of logarithmic CAC scores at follow-up and baseline $(\log [$ CAC (follow-up) +1$]-\log [$ CAC (baseline) + 1]) (19). According to this approach, CAC progression was normally distributed. CAC progression was defined as 1) incident CAC with increase $>0$ Agatston units at last follow-up examination (year 25) among those with CAC = 0 at baseline (year 15) and 2) any CAC progression with increase $>0$ Agatston units until last follow-up examination (year 25).

Missing covariate values were generated using a fully conditional specification multiple imputation method $(20,21)$. The percentage of missing data before imputation was $1.4 \%$ for smoking, $1.1 \%$ for LDL-C, $0.8 \%$ for alcohol, and $<0.2 \%$ for other covariates. Linear regression analysis was performed to estimate unadjusted and multivariable-adjusted association between a 1-SD increment for continuous FG-SD, FG-CV, and FG-ARV with CAC progression using the following models: 1) unadjusted; 2) adjusted for 
age, sex, and race; 3) adjusted for variables in model 2 plus cumulative risk factors such as BMI, current smoker status, milliliters of daily alcohol consumption, systolic blood pressure (SBP), antihypertensive medication use, physical activity, serum creatinine, total cholesterol, and LDL-C; and 4) adjusted for variables in model 3 plus average FG level and FG level change during variability measurement. To confirm whether the FG variability-CAC progression association is independent of FG increment during follow-up, we further analyzed separate models with adjustment for 1) incidence of diabetes and diabetes medication use during variability measurement and 2) FG level at the time of CAC assessment at year 25. We also included adjustment for baseline CAC for any CAC progression. To evaluate the potential influence of incident diabetes during variability measurement, we further investigated the association of FG variability with CAC progression stratified by diabetes status at the time of CAC assessment at year 25 . A two-sided $P$ value $<0.05$ was considered statistically significant. All analyses were conducted using SPSS, version 20 (SPSS, Chicago, IL).

\section{RESULTS}

Demographic and clinical characteristics of study participants according to quartile of FG-CV are presented in Table 1. The baseline FG level was not gradually increased with quartiles of FG-CV. Conversely, greater changes in FG, FG-SD, FG-ARV, and incidence of diabetes were associated with higher FG-CV during 10 years (all $P<0.001$ ). Changes over time for other cardiovascular risk factors

Table 1-Baseline characteristics of 2,256 participants by quartile of FG-CV from 2000-2001 to 2010-2011

\begin{tabular}{|c|c|c|c|c|c|}
\hline & \multicolumn{4}{|c|}{ FG-CV (\%) } & \multirow[b]{2}{*}{$p^{*}$} \\
\hline & Quartile 1 & Quartile 2 & Quartile 3 & Quartile 4 & \\
\hline$N$ & 563 & 564 & 566 & 563 & \\
\hline Age at Y15 (years), mean (SD) & $40.7(3.5)$ & $40.2(3.5)$ & $40.4(3.6)$ & $40.5(3.6)$ & 0.148 \\
\hline Sex, $n$ (\% male) & $252(44.7)$ & $244(43.3)$ & $250(44.2)$ & $253(44.9)$ & 0.949 \\
\hline Race, $n$ (\% Black) & $202(35.8)$ & $232(41.1)$ & 237 (41.9) & $304(54.0)$ & $<0.001$ \\
\hline BMI at $\mathrm{Y} 15\left(\mathrm{~kg} / \mathrm{m}^{2}\right)$, mean (SD) & $28.0(5.8)$ & $28.1(5.8)$ & $27.9(5.6)$ & $30.0(6.6)$ & $<0.001$ \\
\hline $\mathrm{BMI}$ at $\mathrm{Y} 25\left(\mathrm{~kg} / \mathrm{m}^{2}\right)$, mean (SD) & $29.2(6.4)$ & $29.3(6.0)$ & $29.3(6.0)$ & $31.7(7.1)$ & $<0.001$ \\
\hline Current smoker at Y15, $n$ (\%) & $97(17.2)$ & $102(18.1)$ & $98(17.3)$ & $143(25.4)$ & 0.001 \\
\hline Current smoker at Y25, $n(\%)$ & $79(14.0)$ & $79(14.0)$ & 77 (13.6) & $103(18.3)$ & 0.164 \\
\hline Alcohol consumption at Y15 (mL/day), mean (SD) & $9.9(21.0)$ & $10.6(20.6)$ & $10.9(20.4)$ & $11.5(22.3)$ & 0.638 \\
\hline Alcohol consumption at $\mathrm{Y} 25$ (mL/day), mean (SD) & $12.5(24.7)$ & $11.4(18.8)$ & $11.3(18.3)$ & $12.1(21.5)$ & 0.738 \\
\hline SBP at Y15 (mmHg), mean (SD) & $111.5(14.2)$ & $111.1(12.8)$ & $111.2(12.7)$ & $116.9(16.4)$ & $<0.001$ \\
\hline SBP at Y25 (mmHg), mean (SD) & $118.3(15.2)$ & $117.7(15.8)$ & $118.7(14.6)$ & $123.1(18.0)$ & $<0.001$ \\
\hline BP medication use at $\mathrm{Y} 15, n$ (\%) & $32(5.7)$ & $28(5.0)$ & $33(5.8)$ & $63(11.2)$ & $<0.001$ \\
\hline BP medication use at Y25, $n(\%)$ & $117(20.7)$ & $133(23.6)$ & $132(23.3)$ & $238(42.3)$ & $<0.001$ \\
\hline Physical activity at Y15 (exercise units), mean (SD) & $348.2(274.3)$ & $361.1(268.1)$ & $359.0(294.1)$ & $332.6(281.6)$ & 0.300 \\
\hline Physical activity at Y25 (exercise units), mean (SD) & $350.4(284.8)$ & $353.2(269.6)$ & $345.0(283.9)$ & $305.1(264.3)$ & 0.011 \\
\hline Serum creatinine at $\mathrm{Y} 15(\mathrm{mg} / \mathrm{dL})$, mean $(\mathrm{SD})$ & $0.97(0.16)$ & $1.00(0.32)$ & $0.98(0.17)$ & $0.99(0.30)$ & 0.122 \\
\hline Serum creatinine at $\mathrm{Y} 25(\mathrm{mg} / \mathrm{dL})$, mean (SD) & $0.87(0.18)$ & $0.88(0.24)$ & $0.88(0.52)$ & $0.88(0.37)$ & 0.924 \\
\hline Total cholesterol at Y15 (mg/dL), mean (SD) & $186.1(33.6)$ & $184.1(33.4)$ & $185.7(34.7)$ & $186.0(35.9)$ & 0.729 \\
\hline Total cholesterol at Y25 (mg/dL), mean (SD) & $193.6(35.0)$ & $193.6(36.8)$ & $194.2(36.1)$ & $191.6(38.0)$ & 0.648 \\
\hline LDL-C at Y15 (mg/dL), mean (SD) & $114.6(30.2)$ & $112.8(31.7)$ & $114.5(32.1)$ & $113.1(31.4)$ & 0.701 \\
\hline LDL-C at Y25 (mg/dL), mean (SD) & $113.1(30.3)$ & $112.6(33.4)$ & $114.3(32.0)$ & $110.2(34.0)$ & 0.202 \\
\hline FG at $\mathrm{Y} 15(\mathrm{mg} / \mathrm{dL})$, mean $(\mathrm{SD})$ & $87.8(7.7)$ & $84.7(8.7)$ & $81.6(9.9)$ & $89.8(32.0)$ & $<0.001$ \\
\hline FG at $\mathrm{Y} 20(\mathrm{mg} / \mathrm{dL})$, mean (SD) & $91.1(7.6)$ & $92.8(9.1)$ & $94.9(10.9)$ & $113.6(46.6)$ & $<0.001$ \\
\hline FG at $\mathrm{Y} 25(\mathrm{mg} / \mathrm{dL})$, mean (SD) & $90.9(7.8)$ & $92.7(9.8)$ & $94.7(11.1)$ & $121.3(50.6)$ & $<0.001$ \\
\hline Change in FG (mg/dL), mean (SD) & $4.5(2.8)$ & $9.4(3.8)$ & $13.9(5.3)$ & $40.5(43.0)$ & $<0.001$ \\
\hline Incident diabetes by Y25, $n$ (\%) & $13(2.3)$ & $13(2.3)$ & $21(3.7)$ & $101(17.9)$ & $<0.001$ \\
\hline \multicolumn{6}{|l|}{ FG variability } \\
\hline FG-SD (mg/dL), mean (SD) & $3.4(1.2)$ & $6.4(1.0)$ & $9.4(1.4)$ & $26.6(25.7)$ & $<0.001$ \\
\hline FG-CV (\%), mean (SD) & $3.8(1.3)$ & $7.2(0.9)$ & $10.4(1.1)$ & $21.9(12.4)$ & $<0.001$ \\
\hline FG-ARV (mg/dL per year), mean (SD) & $4.2(1.9)$ & $7.6(2.1)$ & $11.0(2.8)$ & $30.5(32.3)$ & $<0.001$ \\
\hline CAC Agatston score at $\mathrm{Y} 15$, median (Q1-Q3) & $0(0-0)$ & $0(0-0)$ & $0(0-0)$ & $0(0-0)$ & 0.656 \\
\hline CAC Agatston score at $\mathrm{Y} 25$, median (Q1-Q3) & $0(0-2.3)$ & $0(0-2.2)$ & $0(0-3.7)$ & $0(0-18.7)$ & 0.002 \\
\hline CAC volume $\left(\mathrm{mm}^{3}\right)$ at $\mathrm{Y} 15$, median (Q1-Q3) & $0(0-0)$ & $0(0-0)$ & $0(0-0)$ & $0(0-0)$ & 0.222 \\
\hline CAC volume $\left(\mathrm{mm}^{3}\right)$ at $\mathrm{Y} 25$, median (Q1-Q3) & $0(0-2.4)$ & $0(0-1.7)$ & $0(0-3.4)$ & $0(0-15.7)$ & 0.002 \\
\hline Any CAC present at Y15, $n$ (\%) & $45(8.0)$ & $50(8.9)$ & $44(7.8)$ & $55(9.8)$ & 0.618 \\
\hline Any CAC present at Y25, $n(\%)$ & $150(26.6)$ & $149(26.4)$ & $162(28.6)$ & $197(35.0)$ & 0.004 \\
\hline
\end{tabular}

$Q$, quartile; $Y$, year. ${ }^{*} P$ value for global test: ANOVA for continuous variables and Pearson $\chi^{2}$ tests for categorical variables. 
such as increasing $\mathrm{BMI}$, systolic $\mathrm{BP}$, and use of BP-lowering medications were associated with higher FG-CV (all $P<$ 0.001). However, age, sex, alcohol consumption, serum creatinine, and cholesterol levels were not significantly different according to quartile of FG-CV. Findings were similar between these characteristics with FG-SD and FG-ARV during 10 years.

CAC progression among participants during 10 years is shown in Supplementary Fig. 2. Participants with baseline CAC at the year 15 examination experienced faster progression of CAC compared with those without baseline CAC (mild CAC progression, $19.3 \%$ vs. $31.4 \%$; moderate CAC progression, $3.0 \%$ vs. $45.4 \%$; and severe CAC progression, $0.2 \%$ vs. $23.2 \%)$. Furthermore, CAC progression was stratified by the quartile of FG-CV (Fig. 1). Findings showed that no significant difference in CAC score was observed at baseline (at year 15); however, after the 5-year follow-up, CAC scores presented a significant difference according to quartile of FG-CV (Q1 vs. Q4, $P=0.030)$ and further increased at year 25 (Q1 vs. Q4, $P<0.001$ ). Similar trends were also observed in FG-SD and FG-ARV (Supplementary Fig. 3). Taken together, CAC progression was gradually increased with quartiles of FG variability during the 10-year follow-up.

The unadjusted and adjusted effect estimates for the association of FG-CV with CAC progression are shown in Table 2. At year 25 , a 1-SD increment in FG-CV showed significant association with progression of CAC as demonstrated as percent change in (CAC +1$)$, with incident CAC 9.1\% $(95 \% \mathrm{Cl} 6.3,11.8)$ and any CAC progression $9.5 \%(95 \% \mathrm{Cl} 6.9$, 12.0) after adjustment for demographics (Table 2, model 2), but this association was attenuated after additional adjustment for cumulative risk factors (Table 2, model 3). Additionally, we observed the association for 1-SD increment in FG-CV across all test results for the final model, model 4, including adjustment for average of FG and change in FG level during variability measurement: incident CAC $5.9 \%(95 \% \mathrm{Cl} 1.0,10.7)$ and any CAC progression $6.7 \%(95 \% \mathrm{Cl} 2.3,11.1)$. The associations between FG-CV with the incident CAC and any CAC progression remained significant after further adjustment for the incidence of diabetes, diabetes medication use (Table 2, model 4A), FG level at year 25 (Table 2, model
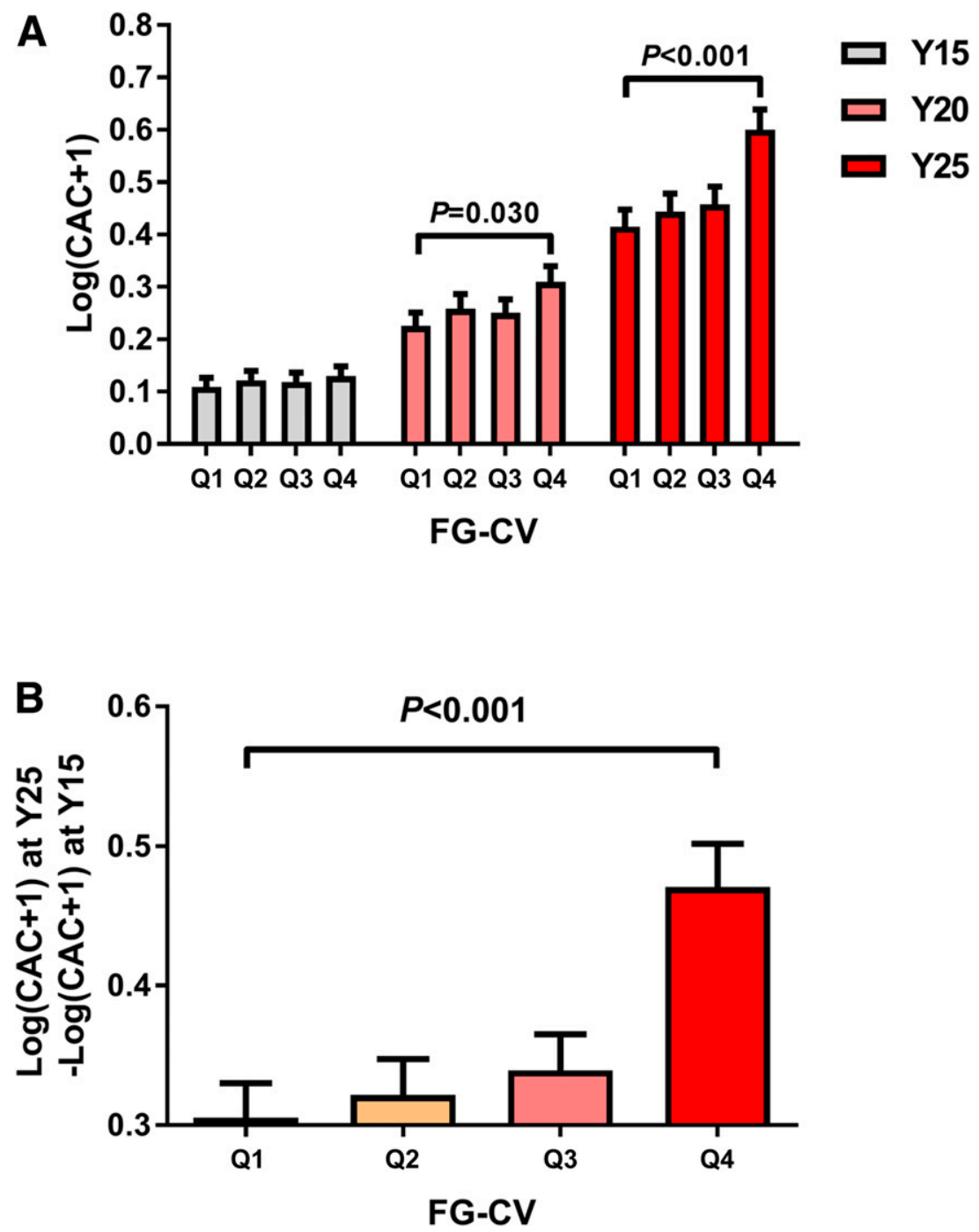

Figure 1-CAC progression according to the quartile of FG-CV A: CAC score at baseline (year 15), 5-year (year 20), and 10-year (year 25) follow-up. B: CAC progression gradually increased with quartiles of FG-CV during the 10-year follow-up. $Q$, quartile; $Y$, year.

4B), and baseline CAC (Table 2, model $4 C)$. A depiction of FG-CV with CAC progression during 10 years is presented in Supplementary Fig. 4. Similar patterns of association between CAC progression with FG-SD and FG-ARV were also observed (Supplementary Tables 1 and 2). Besides, findings were similar between FG variability and CAC progression when we used volume of CAC to estimate CAC progression (Supplementary Tables 3-5).

The associations of FG-CV with the incident $C A C$ and any CAC progression during the 10-year follow-up were stronger among subjects without diabetes than for subjects with diabetes (Supplementary Table 6). This disparate strength of association by diabetes status was not statistically significant (all $P$ for interaction by diabetes status $>0.1$ ). Additionally, neither incident CAC nor any CAC progression was associated with FG-CV among subjects with diabetes after further adjustment for cumulative risk factors, average of FG, and change in FG level during variability measurement. Among subjects without diabetes, however, these associations remained significantly associated (Fig. 2). Similar patterns of association of CAC progression with FG-SD and FG-ARV were also observed stratified by diabetes status. Of note, both FG-SD and FG-ARV were associated with CAC progression among subjects without diabetes even after adjustment for average of FG and change in FG level. A depiction of FG-SD and FG-ARV 
Table 2-Multivariable association of a 1-SD increase in FG-CV with CAC progression over 10 years among individuals in CARDIA

\begin{tabular}{lcc} 
Model & $\begin{array}{c}\text { \% CAC progression per 1-SD } \\
\text { increase in FG-CV }(95 \% \mathrm{Cl})\end{array}$ & $P$ \\
\hline Incident CAC ( $>$ 0 Agatston units), $N=2,062^{*}$ & & \\
Model 1 & $9.3(6.5,12.1)$ & $<0.001$ \\
Model 2 & $9.1(6.3,11.8)$ & $<0.001$ \\
Model 3 & $6.7(4.0,9.4)$ & $<0.001$ \\
Model 4 & $5.9(1.0,10.7)$ & 0.028 \\
Model 4A & $5.3(0.0,10.5)$ & 0.049 \\
Model 4B & $5.9(1.1,10.6)$ & 0.028 \\
Any CAC progression ( $>$ 0 Agatston units), $N=2,256^{+}$ & & \\
Model 1 & $9.6(6.9,12.2)$ & $<0.001$ \\
Model 2 & $9.5(6.9,12.0)$ & $<0.001$ \\
Model 3 & $6.8(4.3,9.3)$ & $<0.001$ \\
Model 4 & $6.7(2.3,11.1)$ & 0.004 \\
Model 4A & $6.0(1.5,10.6)$ & 0.012 \\
Model 4B & $6.8(2.3,11.4)$ & 0.004 \\
Model 4C & $6.8(2.2,11.4)$ & 0.004 \\
\hline
\end{tabular}

A 1-SD unit increment in FG-CV is 9.3\%. Model 1 , no adjustment; model 2 , adjustment for age, sex and race; model 3, adjustment for variables in model 2 plus BMI, current smoker status (yes/no), milliliters of daily alcohol consumption, SBP, antihypertensive medication use (yes/no), physical activity, serum creatinine, total cholesterol, and LDL-C; model 4, adjustment for variables in mode 3 plus average of $\mathrm{FG}$ and change in $\mathrm{FG}$ level during variability measurement; model $4 \mathrm{~A}$, adjustment for variables in model 4 plus incidence of diabetes and diabetes medication use; model $4 B$ adjustment for variables in model 4 plus FG level at year 25 ; model $4 C$, for individuals with any CAC progression, adjustment for variables in model 4 plus baseline CAC. *Evaluated only among individuals without baseline CAC at the year 15 examination. $+C A C$ progression was calculated as the difference of logarithmic CAC score at follow-up and baseline (log[CAC (follow-up) +1] $\log [$ CAC (baseline) +1$]$ ).

with CAC progression at year 25 is presented in Supplementary Figs. 5 and 6 . Subgroup analysis showed no significant difference stratified by sex category (all $P$ for interaction $>0.1$ ) (Supplementary Table 7), but stronger association of FG-SD or FG-ARV with incident CAC and any CAC progression was observed in the White subgroup ( $P$ for interaction $<0.05$ ) (Supplementary Table 8).

\section{CONCLUSIONS}

In this prospective cohort study with 10 years of follow-up, we demonstrate for the first time that greater FG variability during young adulthood was independently associated with greater CAC progression by middle age. This association was observed after adjustment for demographic and cardiovascular risk factors, average FG level, change in FG level during variability measurement, diabetes incidence, and medication use. We also show that FG variability, as determined by $C V, S D$, and $A R V$, was more strongly associated with CAC progression in subjects without diabetes than in those with diabetes. This was possibly attributable to the use of diabetes medication and the development of diabetes weakening the association between glycemic variability and CAC progression. Subjects with diabetes experience greater CAC progression than those without diabetes (22). In addition, diabetes medication use disrupts the natural course of glycemic variability and may alter any association of glycemic variability with CAC progression $(23,24)$.

Several potential pathophysiological mechanisms may contribute to the association between glycemic variability and CAC progression. Firstly, greater glycemic fluctuation has been shown to induce overproduction of superoxide, reduce nitric oxide availability, trigger activation of monocytes and macrophages, and increase inflammatory cytokine generation, which result in endothelial damage and vascular remodeling (25-29). These changes on vascular structure and function are common findings in CAC development. Secondly, transient hyperglycemia may induce alkaline phosphatase activation and osteogenic changes in vascular smooth muscle cells, which are involved in the onset or progression of CAC (30). Thirdly, greater long-term glycemic variability may induce cellular metabolic memory and increase insulin resistance (31), and these processes may contribute to the increased risk of CAC (32). Lastly, increased glycemic variability may be a marker of poor quality of life, lack of social support, and other comorbidities (33). Consistent with previous studies, we found that individuals with greater FG variability tend to be less physically active and had higher BMI and SBP.

Emerging evidence demonstrates that glycemic variability may be an important predictor of stoke, retinopathy, and other cardiovascular complications among older individuals with diabetes $(12,13,31)$. However, few studies have specifically investigated the relationship between FG variability and CAC (a predictor of macrovascular complications) in young adults and adults in early middle age. Prior epidemiological and clinical studies of glycaemia and CAC have reported conflicting results. No association between impaired FG and CAC was reported after adjustment for confounding factors in the Framingham Heart Study (34). In contrast, findings from the Heinz Nixdorf Recall Study showed significant association between impaired $F G$ and CAC (35). In CARDIA, higher $\mathrm{HbA}_{1 \mathrm{c}}$ was also associated with advanced CAC progression (36). $\mathrm{HbA}_{1 \mathrm{c}}$ level reflects average glucose over a period of 8-12 weeks, but it fails to capture real-time glycemic fluctuation (37). Recent evidence indicated that long-term visit-to-visit FG variability was a better predictor than mean $\mathrm{HbA}_{1 \mathrm{c}}$ for assessing the risk of future development of micro- and macrovascular complications (31). Moreover, results from the Veterans Affairs Diabetes Trial showed that variability of FG other than $\mathrm{HbA}_{1 \mathrm{c}}$ level was associated with cardiovascular disease after adjustment for multiple baseline covariates, suggesting that FG variability may be a more sensitive indicator in regard to potential cardiovascular complications (11). Therefore, it is important to elucidate the association between glycemic variability and CAC development. In the current study, we define long-term glycemic variability by estimating $C V, S D$, and $A R V$ for $F G$ and provide further 
A

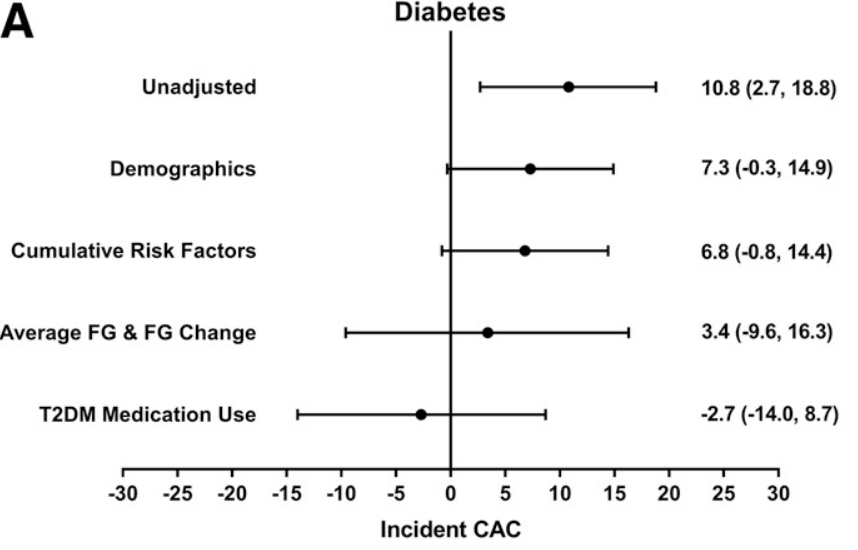

C

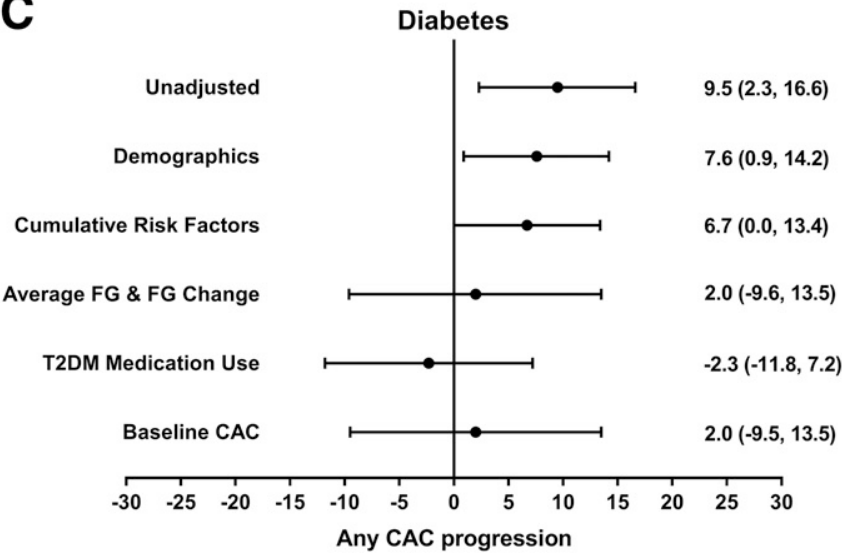

B

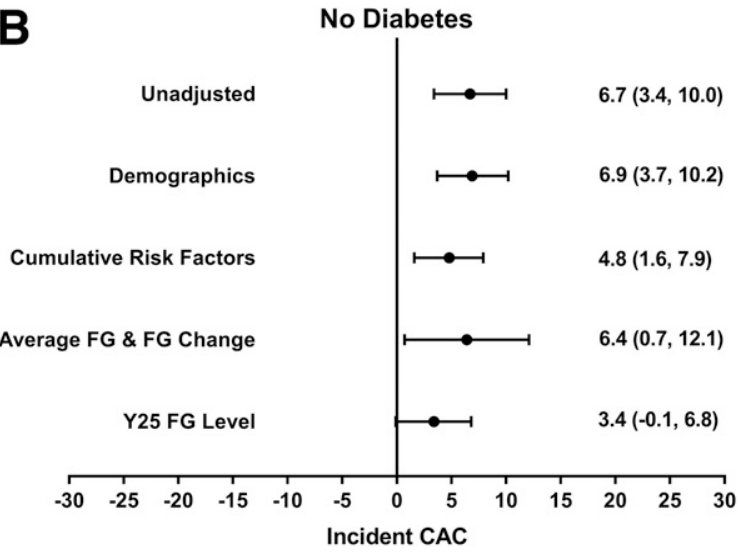

D

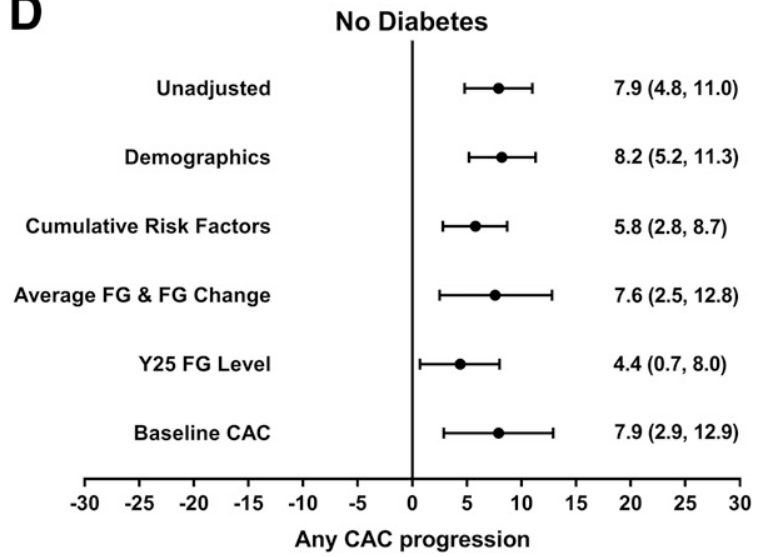

Figure 2-Forest plot of the association between a 1-SD unit increment in FG-CV and percent progression of incident CAC among individuals with diabetes $(A)$ or without diabetes $(B)$ and any CAC progression among individuals with diabetes $(C)$ or without diabetes $(D)$. Model adjustment: demographics - age, sex, and race; cumulative risk factors-demographics plus BMI, current smoker status, milliliters of daily alcohol consumption, SBP, antihypertensive medication use, physical activity, serum creatinine, total cholesterol, and LDL-C; average FG \& FG change-cumulative risk factors plus average FG level and change in FG level during variability measurement; T2DM medication use-average FG \& FG change plus use of medication for type 2 diabetes; Y25 FG level-average FG \& FG change plus FG level at year 25; baseline CAC-average FG \& FG change plus baseline CAC.

evidence that greater FG variability during young adulthood leads to greater CAC progression by middle age.

The strengths of our study include its status as a prospective study related to subclinical coronary artery disease among young adults and adults in early middle age during 10 years of follow-up and the relatively large sample size, standardized data collection protocols, and rigorous quality control. Some potential limitations should be considered. Assessment of CAC began at the year 15 examination, so participants were not included in these analyses due to missing CAC score at years 15 and 25. Exclusion of these participants may have affected our findings, but the retention of CARDIA participants remained high. Interscan variability may have a potential effect on the change of CAC scores, but previous studies in
CARDIA showed high between-reader and within-reader reproducibility $(17,38)$. FG measures were collected every 5 years and may not reflect daily glucose fluctuation, traditionally assessed among individuals. $\mathrm{HbA}_{1 \mathrm{c}}$ and 2-h glucose levels were not measured frequently enough during CARDIA follow-up to estimate variability for these measures and their association with CAC progression. Lastly, this was an observational study, and other potential residual and unknown confounding variables may be present.

In conclusion, we observed that greater individual variability in FG during young adulthood was associated with greater CAC progression by middle age. These results suggest that efforts to achieve normal consistent levels of glucose very early in life may reduce the risk for future
CAC and other subclinical coronary artery diseases.

Acknowledgments. The authors thank the other investigators, the staff, and the participants of CARDIA for their important contributions.

Funding. CARDIA is conducted and supported by the National Heart, Lung, and Blood Institute (NHLBI) in collaboration with the University of Alabama at Birmingham (HHSN268201300025C and HHSN268201300026C), Northwestern University (HHSN268201300027C), University of Minnesota (HHSN268201300028C), Kaiser Foundation Research Institute (HHSN268201300029C), and Johns Hopkins University School of Medicine (HHSN268200900041C). CARDIA is also partially supported by the Intramural Research Program of the National Institute on Aging (NIA) and an intra-agency agreement between NIA and NHLBI (AG0005). Additional support was provided by the National Natural Science Foundation of China (81700258 to F.H.; 31671025, 31771099, and 81871504 to C.O.; and U1501222, 31771060, and 81971765 to M.C.). 
Duality of Interest. No potential conflicts of interest relevant to this article were reported. Author Contributions. W.F., C.O., F.H., and $M . C$. conceived the research idea and designed the analysis. W.F., C.O., and F.H. analyzed and interpreted the data. M.C. advised on statistical analysis methods. W.F. drafted the manuscript. Z.L., W.G., X.F., F.Z., and K.Z. contributed to the discussion. W.F., Z.L., C.O., F.H., and M.C. provided critical revision of the manuscript for important intellectual content. W.F., Z.L., W.G., X.F., F.Z., K.Z., C.O., F.H., and M.C. reviewed and approved the final manuscript. M.C. is the guarantor of this work and, as such, had full access to all the data in the study and takes responsibility for the integrity of the data and the accuracy of the data analysis.

\section{References}

1. Sarwar N, Gao P, Seshasai SR, et al.; Emerging Risk Factors Collaboration. Diabetes mellitus, fasting blood glucose concentration, and risk of vascular disease: a collaborative meta-analysis of 102 prospective studies [published correction appears in Lancet 2010;376:958]. Lancet 2010; 375:2215-2222

2. Cavender MA, Steg PG, Smith SC Jr., et al.; REACH Registry Investigators. Impact of diabetes mellitus on hospitalization for heart failure, cardiovascular events, and death: outcomes at 4 years from the Reduction of Atherothrombosis for Continued Health (REACH) registry. Circulation 2015;132:923-931

3. Kannel WB, McGee DL. Diabetes and glucose tolerance as risk factors for cardiovascular disease: the Framingham Study. Diabetes Care 1979;2:120-126

4. Yeboah J, McClelland RL, Polonsky TS, et al. Comparison of novel risk markers for improvement in cardiovascular risk assessment in intermediaterisk individuals. JAMA 2012;308:788-795

5. Shaw L, Raggi P, Schisterman E, Berman DS, Callister TQ. Prognostic value of cardiac risk factors and coronary artery calcium screening for allcause mortality. Radiology 2003;228:826-833

6. Pletcher MJ, Tice JA, Pignone M, Browner WS. Using the coronary artery calcium score to predict coronary heart disease events: a systematic review and meta-analysis. Arch Intern Med 2004; 164:1285-1292

7. Greenland $\mathrm{P}$, LaBree L, Azen SP, Doherty TM, Detrano RC. Coronary artery calcium score combined with Framingham score for risk prediction in asymptomatic individuals. JAMA 2004;291: 210-215

8. American Diabetes Association. 9. Cardiovascular disease and risk management: Standards of Medical Care in Diabetes-2018. Diabetes Care 2018;41(Suppl. 1):S86-S104

9. Fox CS, Golden SH, Anderson C, et al.; American Heart Association Diabetes Committee of the Council on Lifestyle and Cardiometabolic Health, Council on Clinical Cardiology, Council on Cardiovascular and Stroke Nursing, Council on Cardiovascular Surgery and Anesthesia, Council on Quality of Care and Outcomes Research, and the American Diabetes Association. Update on prevention of cardiovascular disease in adults with type 2 diabetes mellitus in light of recent evidence: a scientific statement from the American Heart Association and the American Diabetes Association. Circulation 2015;132:691-718
10. Wang A, Liu $X, X u J$, et al. Visit-to-visit variability of fasting plasma glucose and the risk of cardiovascular disease and all-cause mortality in the general population. J Am Heart Assoc 2017:6:e006757

11. Zhou JJ, Schwenke DC, Bahn G, Reaven P; VADT Investigators. Glycemic variation and cardiovascular risk in the Veterans Affairs Diabetes Trial. Diabetes Care 2018;41:2187-2194

12. Lin $\mathrm{CC}$, Yang $\mathrm{CP}, \mathrm{Li} \mathrm{Cl}$, et al. Visit-to-visit variability of fasting plasma glucose as predictor of ischemic stroke: competing risk analysis in a national cohort of Taiwan Diabetes Study. BMC Med 2014;12:165

13. Hirakawa $Y$, Arima $H$, Zoungas $S$, et al. Impact of visit-to-visit glycemic variability on the risks of macrovascular and microvascular events and allcause mortality in type 2 diabetes: the ADVANCE trial. Diabetes Care 2014;37:2359-2365

14. Friedman GD, Cutter GR, Donahue RP, et al. CARDIA: study design, recruitment, and some characteristics of the examined subjects. J Clin Epidemiol 1988;41:1105-1116

15. Bancks MP, Carnethon MR, Jacobs DR Jr., et al. Fasting glucose variability in young adulthood and cognitive function in middle age: the Coronary Artery Risk Development in Young Adults (CARDIA) study. Diabetes Care 2018;41 2579-2585

16. Kershaw KN, Robinson WR, Gordon-Larsen $P$, et al. Association of changes in neighborhoodlevel racial residential segregation with changes in blood pressure among black adults: the CARDIA study. JAMA Intern Med 2017;177:996-1002 17. Carr JJ, Nelson JC, Wong ND, et al. Calcified coronary artery plaque measurement with cardiac CT in population-based studies: standardized protocol of Multi-Ethnic Study of Atherosclerosis (MESA) and Coronary Artery Risk Development in Young Adults (CARDIA) study. Radiology 2005 234:35-43

18. Agatston AS, Janowitz WR, Hildner FJ Zusmer NR, Viamonte M Jr., Detrano R. Quantification of coronary artery calcium using ultrafast computed tomography. J Am Coll Cardiol 1990;15:827-832

19. Mahabadi AA, Lehmann N, Kälsch $\mathrm{H}$, et al. Association of epicardial adipose tissue with progression of coronary artery calcification is more pronounced in the early phase of atherosclerosis: results from the Heinz Nixdorf recall study. JACC Cardiovasc Imaging 2014;7:909-916 20. Lee KJ, Carlin JB. Multiple imputation for missing data: fully conditional specification versus multivariate normal imputation. Am J Epidemiol 2010;171:624-632

21. van Buuren S. Multiple imputation of discrete and continuous data by fully conditional specification. Stat Methods Med Res 2007;16:219-242 22. Kronmal RA, McClelland RL, Detrano R, et al. Risk factors for the progression of coronary artery calcification in asymptomatic subjects: results from the Multi-Ethnic Study of Atherosclerosis (MESA). Circulation 2007;115:27222730

23. Li TC, Yang CP, Tseng ST, et al. Visit-to-visit variations in fasting plasma glucose and $\mathrm{HbA}_{1 \mathrm{c}}$ associated with an increased risk of Alzheimer disease: Taiwan Diabetes Study. Diabetes Care 2017;40:1210-1217

24. Bancks MP, Carson AP, Lewis CE, et al. Fasting glucose variability in young adulthood and incident diabetes, cardiovascular disease and all-cause mortality. Diabetologia 2019;62: 1366-1374

25. Ceriello A, Esposito K, Piconi L, et al. Oscillating glucose is more deleterious to endothelial function and oxidative stress than mean glucose in normal and type 2 diabetic patients. Diabetes 2008;57:1349-1354

26. Ceriello A, Ihnat MA. 'Glycaemic variability': a new therapeutic challenge in diabetes and the critical care setting. Diabet Med 2010;27:862867

27. Nishikawa T, Edelstein D, Du XL, et al. Normalizing mitochondrial superoxide production blocks three pathways of hyperglycaemic damage. Nature 2000;404:787-790

28. Keating ST, El-Osta A. Glycemic memories and the epigenetic component of diabetic nephropathy. Curr Diab Rep 2013;13:574-581

29. Monnier L, Mas E, Ginet C, et al. Activation of oxidative stress by acute glucose fluctuations compared with sustained chronic hyperglycemia in patients with type 2 diabetes. JAMA 2006;295: 1681-1687

30. Terao Y, Satomi-Kobayashi S, Hirata K, Rikitake $Y$. Involvement of Rho-associated protein kinase (ROCK) and bone morphogenetic proteinbinding endothelial cell precursor-derived regulator (BMPER) in high glucose-increased alkaline phosphatase expression and activity in human coronary artery smooth muscle cells. Cardiovasc Diabetol 2015;14:104

31. Cardoso CRL, Leite NC, Moram CBM, Salles GF. Long-term visit-to-visit glycemic variability as predictor of micro- and macrovascular complications in patients with type 2 diabetes: the Rio de Janeiro Type 2 Diabetes Cohort Study. Cardiovasc Diabetol 2018;17:33

32. Schauer IE, Snell-Bergeon JK, Bergman BC, et al. Insulin resistance, defective insulinmediated fatty acid suppression, and coronary artery calcification in subjects with and without type 1 diabetes: the CACTI study. Diabetes 2011 60:306-314

33. Gorst C, Kwok CS, Aslam S, et al. Long-term glycemic variability and risk of adverse outcomes: a systematic review and meta-analysis. Diabetes Care 2015;38:2354-2369

34. Rutter MK, Massaro JM, Hoffmann U, O'Donnell CJ, Fox CS. Fasting glucose, obesity and coronary artery calcification in communitybased people without diabetes. Diabetes Care 2012:35:1944-1950

35. Moebus S, Stang A, Möhlenkamp S, et al. Heinz Nixdorf Recall Study Group. Association of impaired fasting glucose and coronary artery calcification as a marker of subclinical atherosclerosis in a population-based cohort-results of the Heinz Nixdorf Recall Study. Diabetologia 2009;52:81-89

36. Carson AP, Steffes MW, Carr JJ, et al. Hemoglobin $A_{1 c}$ and the progression of coronary artery calcification among adults without diabetes. Diabetes Care 2015;38:66-71

37. Brownlee M, Hirsch IB. Glycemic variability: a hemoglobin A1c-independent risk factor for diabetic complications. JAMA 2006;295:1707-1708 38. Yan LL, Liu K, Daviglus ML, et al. Education, 15-year risk factor progression, and coronary artery calcium in young adulthood and early middle age: the Coronary Artery Risk Development in Young Adults study. JAMA 2006;295:1793-1800 